Supporting Information

\title{
A Camel Nose-Inspired Highly Durable Neuromorphic Humidity Sensor with Water Source Locating Capability
}

Caicong Li, ${ }^{\dagger \neq \&}$ Jie Liu, ${ }^{\dagger+z \&}$ Hailong Peng, ${ }^{*} \&$ Yuan Sui, ${ }^{\dagger+}$ Jian Song, ${ }^{*} *$ Yang Liu, ${ }^{\dagger \neq}$ Wei Huang, ${ }^{\dagger z}$ Xiaowei Chen, ${ }^{\dagger *}$ Jinghui Shen, ${ }^{\dagger+}{ }^{+}$Yao Ling, ${ }^{\dagger}{ }^{+}$Chongyu Huang, ${ }^{\dagger+\perp}$ Youwei Hong, ${ }^{\nabla}$ Weiguo Huang ${ }^{\dagger}+1 *$

$\uparrow$ State Key Laboratory of Structural Chemistry, Fujian Institute of Research on the Structure of Matter, Chinese Academy of Sciences. 155 Yangqiao West Road, Fuzhou, Fujian 350002, P. R. China.

\$ Fujian Science \& Technology Innovation Laboratory for Optoelectronic Information of China, Fuzhou, Fujian 350002, P. R. China.

z College of Chemistry and Materials Science, Fujian Normal University, Fuzhou, Fujian 350002, P. R. China.

\# School of Resources, Environmental, and Chemical Engineering, Nanchang University Nanchang 330031, P. R. China. 
$\S$ Science and Technology on Microsystem Laboratory, Shanghai Institute of Microsystem and Information Technology, Chinese Academy of Sciences, Shanghai 200050, P. R. China.

※ College of Chemistry, Fuzhou University, Fuzhou 350108, P. R. China.

$\perp$ University of Chinese Academy of Sciences, 19A Yuquan Road, Beijing 100049, P. R. China.

$\nabla$ Key Lab of Urban Environment and Health, Institute of Urban Environment, Chinese Academy of Sciences, Xiamen, 361021, China.

Noncovalent Interaction (NCI) Analysis: ${ }^{1}$ Based on a model developed by Yang, the quantum-mechanical electron density, $\rho$, and the reduced density gradient, $s(s=$ $\left.1 /\left(2\left(3 \pi^{2}\right)^{1 / 3}\right)|\nabla \rho| / \rho^{4 / 3}\right)$, are the two fundamental quantities for describing the deviation from a homogeneous electron distribution. Covalent bonding and noncovalent bonding could be well distinguished from the plot of $s$ versus $\rho$. In detail, plot pattern of covalent bond shows small electron density and large reduced gradient corresponding to the exponentially decaying tail regions (far from the nuclei) of the electron density, and large electron density and low reduced gradient corresponding to the covalent bond. A saddle point in the electron density (bond critical points, $s=0$ ) is the characteristic of covalent bonds. Whereas, noncovalent interactions could be identified as spike regions with low electron density and low reduced gradient.

To further discriminate between different type of noncovalent interactions (such as hydrogen bonding, van der Waals force, and steric crowding), the sign of the Laplacian of the density, $\operatorname{sign}\left(\lambda_{2}\right) \rho$, is used. A large and negative $\operatorname{sign}\left(\lambda_{2}\right) \rho$ indicates attractive interactions (e.g., dipole-dipole and hydrogen bonding), a large and positive $\operatorname{sign}\left(\lambda_{2}\right) \rho$ suggests nonbonding interaction (e.g., steric crowding). Whereas van der Waals force 
normally manifests itself as a small negative $\operatorname{sign}\left(\lambda_{2}\right) \rho$ value near zero.

Density Functional Theory (DFT) calculations: Geometry optimizations and frequency analyses were implemented by ORCA 4.2.1 program $^{2}$ using dispersion corrected methods B3LYP-D3 ${ }^{3}$ (B3LYP with Grimme's DFT-D3 correction) in conjunction with def2-TZVP basis set. ${ }^{4}$

Moisture content change in industrial exhaust by water drop injection: The full evaporation of $0.2 \mathrm{~mL}$ water drop would lead to $0.335 \mathrm{~L}$ moisture at $95{ }^{\circ} \mathrm{C}$ according to the following equation:

$$
V=\frac{v R T}{M P}
$$

Where $v$ is $0.2 \mathrm{~mL}, R$ is gas constant, $T$ is temperature, $M$ is molecular weight and $P$ is standard atmospheric pressure.

The total volume of the combustion cell as well as the exhaust pipe is around $8 \mathrm{~L}$, therefore, $0.335 \mathrm{~L}$ moisture accounts for $4.19 \%$ total volume.

Concentrations of individual industrial exhaust components: A online-vapor analysis system using a custom-built setup for chip-based electrochemistry-mass spectrometry (chip EC-MS) indicates the exhaust containing a rough concentration of $5.36 \%, 72.7 \%, 17.2 \%, 1.87 \%, 2.88 \%, 0.0088 \%$ and $0.0423 \% \mathrm{H}_{2} \mathrm{O}, \mathrm{N}_{2}, \mathrm{O}_{2}, \mathrm{Ar}, \mathrm{CO}_{2}$, $\mathrm{NO}_{2}$ and $\mathrm{N}_{2} \mathrm{O}_{3}$, respectively.

Fabrication of sensor array: A mixture solution containing (pentafluorophenyl acrylate) (pPFPA), branched polyethylenimine ( $b$ PEI) and poly (methyl methacrylate) (PMMA) with a ratio of 120:32: 152 in toluene/chloroform $(v / v=1 / 19)$ is drop-casted on a thoroughly cleaned Indium tin oxide coated polyethylene terephthalate (PET) substrate or on a silicon wafer, and dried slowly in $\mathrm{N}_{2}$. The resulting film is annealed at $60{ }^{\circ} \mathrm{C}$ for one hour to promote the crosslinking between pPFPA and $b$ PEI and then soaked in chloroform to completely remove PMMA, giving rise to a porous film $\mathrm{F}_{120} \mathrm{E}_{32} \mathrm{M}_{152} \mathrm{P}$, which is then decorated with zwitterions by being soaked in 3-((3aminopropyl)dimethylammonio)propane-1-sulfonate (ADPS). Finally, gold electrodes 
are deposited on the $\mathrm{F}_{120} \mathrm{E}_{32} \mathrm{M}_{152}$-PA film through a shadow mask to form a $13 \times 13$ capacitor sensor array. The dimension of the whole array and each pixel is around 6.0 $\mathrm{cm} \times 6.0 \mathrm{~cm}$ and $2.5 \mathrm{~mm} \times 2.5 \mathrm{~mm}$, respectively.

Measurement of sensor array for perceiving the trajectory of the fingertips: To perceive the trajectory of the fingertip, we manually probe the top electrode one-by-one to acquire the capacitance of each pixel in a scanning mode (from left to right, and from top to bottom) while moving the fingertip. Note that we intentionally move the fingertip at a low speed, so as that the scanning measurement could catch up with the fingertip motion. After we obtained capacitance of all pixels, a heatmap reflecting the capacitance variation and fingertip trajectory across the array is generated by data processing software. Though all pixels were not measured simultaneously, the validity of the experimental results still holds based on following reasons: 1) the perceived trajectory shown in the heatmap is well consistent with the actual path of fingertip movement. 2) Although the slow fingertip motion could cause surface moisture diffusion and affect other pixels, the capacitance change of the main sensing pixel is significantly larger than that of the surrounding ones due to the capacitance decays exponentially with the distance (see Figure S7b). Therefore, signal interference between array pixels is minimized and the trajectory of finger movements can be precisely detected.

Detailed information about the commercial humidity sensor: The commercial humidity sensor (from Jian Da Ren Ke company, Model: COS-03-05 USB) is able to measure temperature and humidity simultaneously. In detail, the temperature is measured by a thermal resistor. The relative humidity is measured by a humidity responsive capacitor. Both the thermal resistor and the humidity responsive capacitor are integrated into a chip.

Calculation of binding constant $(\mathbf{K})$ : The binding constant $(K)$ is calculated according to the following equation : 5

$$
K=e^{\frac{-\Delta G}{R T}}
$$


Where $\Delta G$ is the binding energy, $R$ is the molar gas constant, and $T$ is the temperature in Kelvin.

Simulation of water source for locating experiments: To simulate the water source, a moisture pulse generator with high purity $\mathrm{N}_{2}$ as a moisture carrier is built. As shown in Scheme S2, $\mathrm{N}_{2}$ is bubbled underneath of the water surface, generating moisture pulse with a high humidity level $(\sim 90 \%)$ and a given pulse width by switching the valve. Alternatively, moisture pulses with a high humidity level could also be generated by the human mouth.

\section{Signal conditioning circuit for the sensor and capacitance measurement:}

The signal conditioning circuit is shown in Figure S6. The Voltage $V_{D E V}$ increases when the device is charged. Bipolar junction transistor in 555-timer-rated chip would be turned on when $V_{D E V}>2 / 3 V_{C C}$. The charging time of device is:

$$
t_{P H}=\left(R_{1}+R_{2}\right) C \ln 2
$$

Then the device is in discharge status until $V_{D E V}<1 / 3 V_{C C} . \underline{V}_{\text {out }}$ changes from a logic high to a logic low during this period. The discharging time of our device is:

$$
t_{P L}=R_{2} C \ln 2
$$

A square wave is then obtained and the frequency is:

$$
f=\frac{1}{t_{P H}+t_{P L}}
$$

And the capacitance of the device could be calculated by:

$$
C=1.44 /\left(R_{1}+2 R_{2}\right) f
$$

The capacitor sensor on the arm is wired to the signal conditioning circuit (Figure S6e) which is held in hand by the runner. The circuit in-situ measures and records the capacitance change during running and resting period (Figure S6c-d). The data is then extracted and plotted as in Figure 6a and 6b.

For the sensor array used for location determination, STM32 is used as the main controller of the whole system for data collection and all the calculation mentioned above. Figure S6f is the simplified view of the signal conditioning circuit of capacitive 
sensor array to determine the water source location. Data from sensor 1-4 is processed by STM32 and then displayed on LCD. Fitting these data in to Figure 8 and equation (2) gives rise to the water source location.

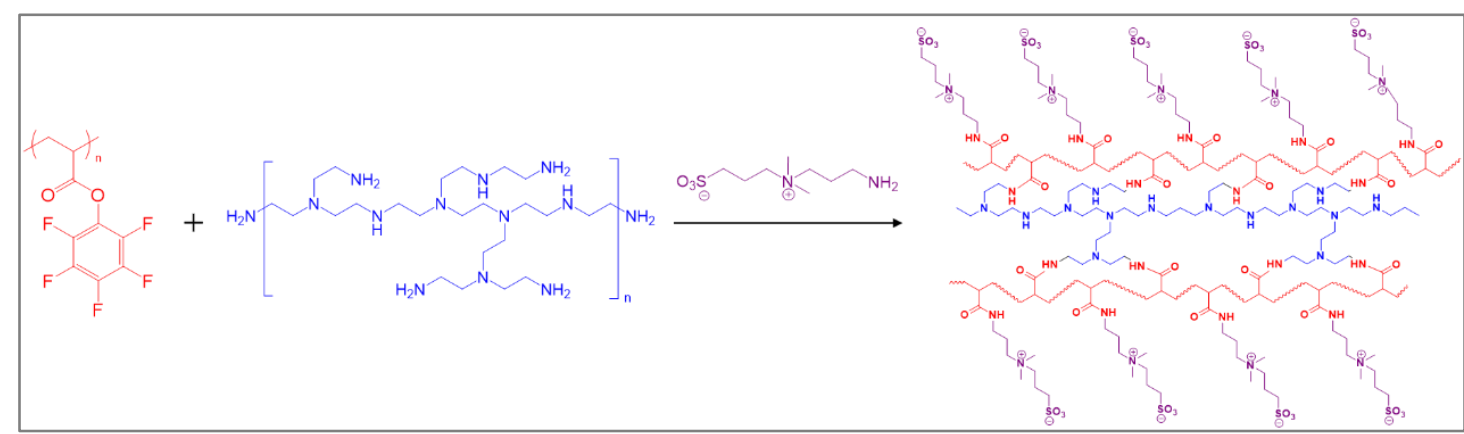

Scheme S1. Crosslinking between pPFPA and $b$ PEI, and ADPS decoration of crosslinked network.

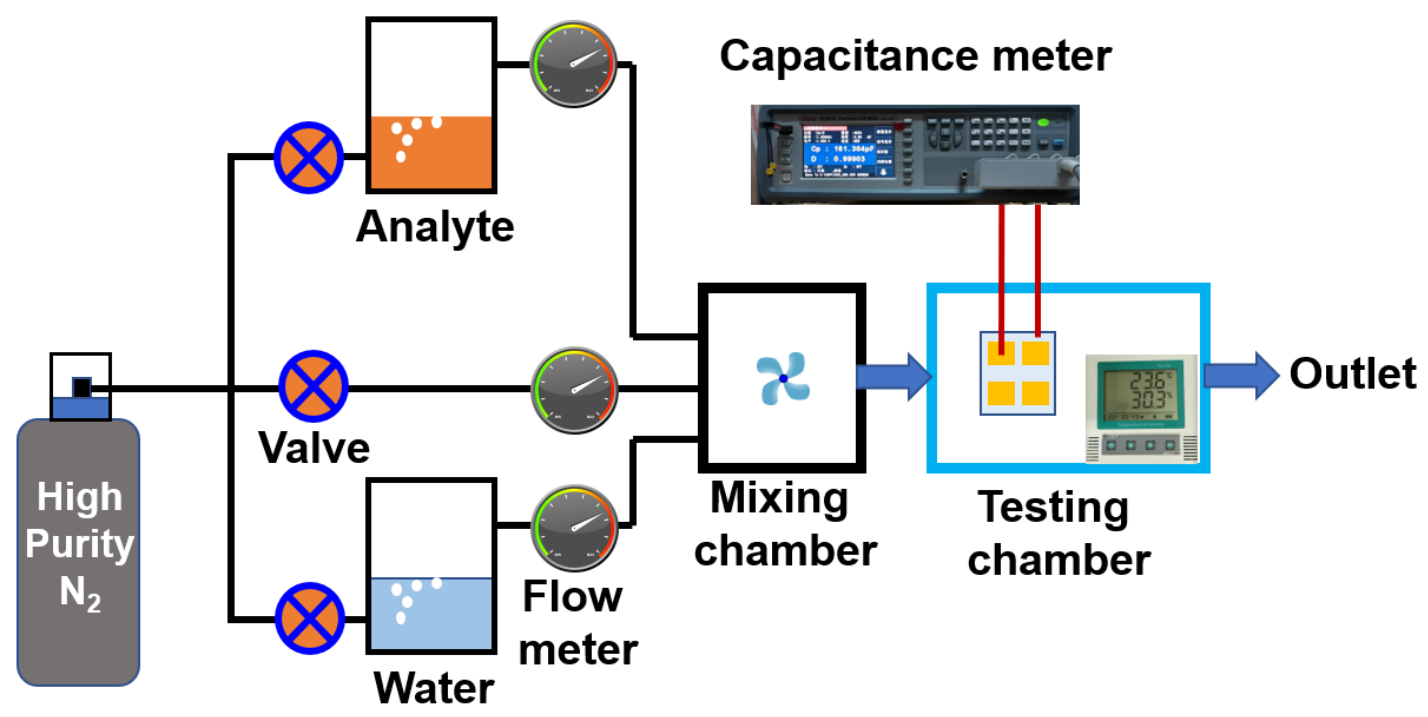

Scheme S2. Schematical illustration of vapor concentration control and testing equipment.

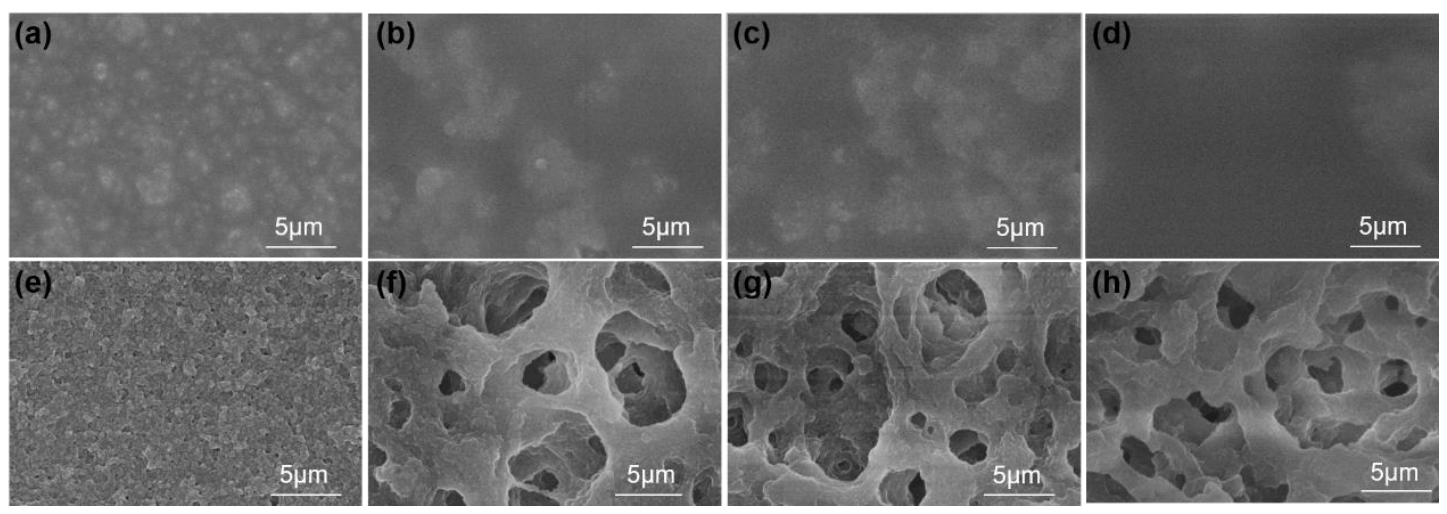


Figure S1. SEM images of (a) $\mathrm{F}_{120} \mathrm{E}_{32} \mathrm{M}_{0}$ and (e) $\mathrm{F}_{120} \mathrm{E}_{32} \mathrm{M}_{0}-\mathrm{P}$, (b) $\mathrm{F}_{120} \mathrm{E}_{32} \mathrm{M}_{38}$ and (f) $\mathrm{F}_{120} \mathrm{E}_{32} \mathrm{M}_{38}-\mathrm{P}$, (c) $\mathrm{F}_{120} \mathrm{E}_{32} \mathrm{M}_{76}$ and (g) $\mathrm{F}_{120} \mathrm{E}_{32} \mathrm{M}_{76}-\mathrm{P}$, (d) $\mathrm{F}_{120} \mathrm{E}_{32} \mathrm{M}_{152}$ and (h) $\mathrm{F}_{120} \mathrm{E}_{32} \mathrm{M}_{152}-$ $\mathrm{P}$. Note that the roughness increase in (e) is probably due to the dissolving of uncrosslinked pPFPA and $b$ PEI in the film.

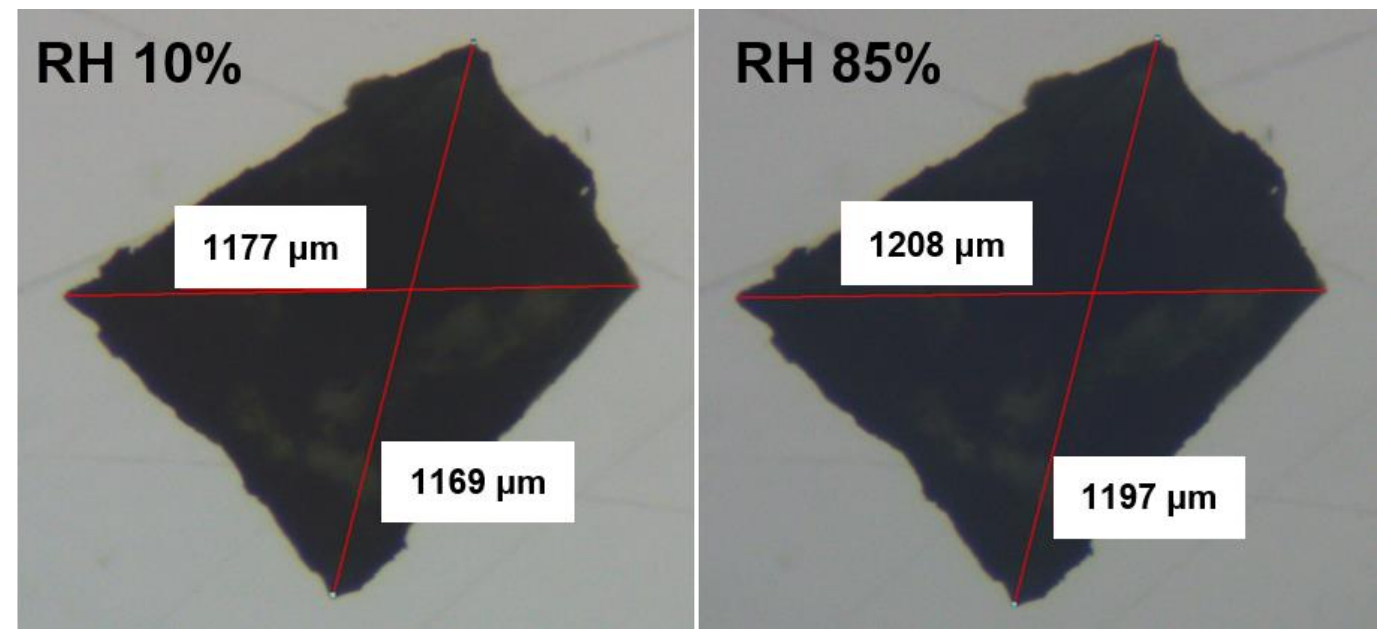

Figure S2. The dimension of zwitterionic porous film at $10 \%$ and $85 \% \mathrm{RH}$, respectively. To demonstrate the air is squeezed out by the moisture swelling process, we quantitatively characterize the density difference of the zwitterionic porous film before and after being exposed to a high humidity level. Here, we hypothesize an isotropic swelling across the film at all dimensions. By carefully measuring the size and mass of the film at $10 \%$ and $85 \% \mathrm{RH}$, respectively, we obtain a volume swelling ratio $\left(V_{S}\right)$ of 1.08 and a mass swelling ratio $(M s)$ of 1.262 . Dividing $M s$ by $V s$ leads to $\mathrm{d}_{85 \%} / \mathrm{d}_{10 \%}$ of 1.17. where $\mathrm{d}_{10 \%}$ and $\mathrm{d}_{85 \%}$ is the film density at $10 \%$ and $85 \% \mathrm{RH}$, respectively, implying that the film density is higher at $85 \% \mathrm{RH}$ than at $10 \%$. This result well agrees with the expectation that a higher density appears at a higher RH due to the squeezing out of air and gaining of water in the film. 

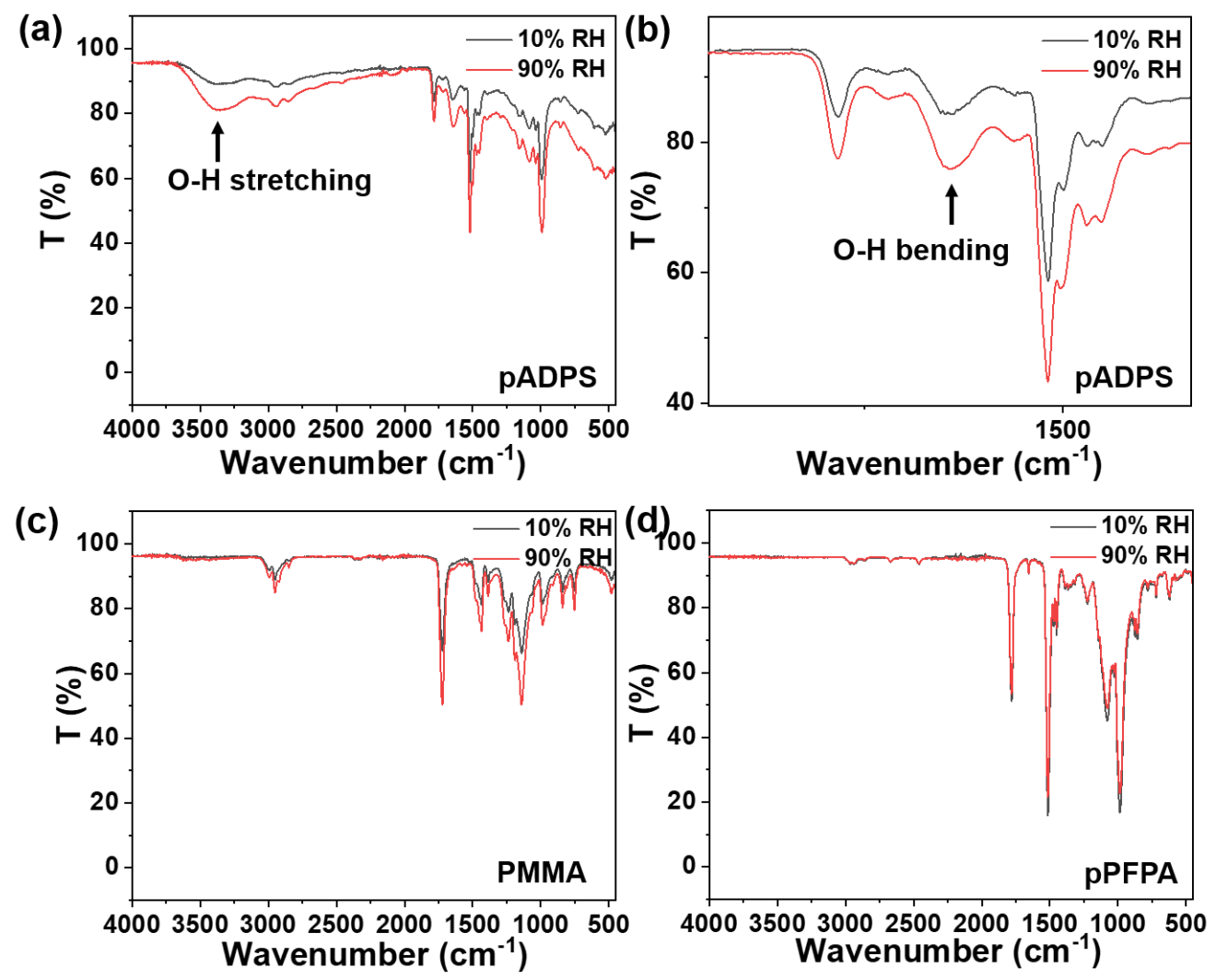

Figure S3. Attenuated Total Reflection (ATR) characterization of (a-b) pADPS, (c) PMMA, and (d) pPFPA at 10\% and 90\% RH, respectively. (b) is the enlarged version of (a) from 1300 to $1900 \mathrm{~cm}^{-1}$. 

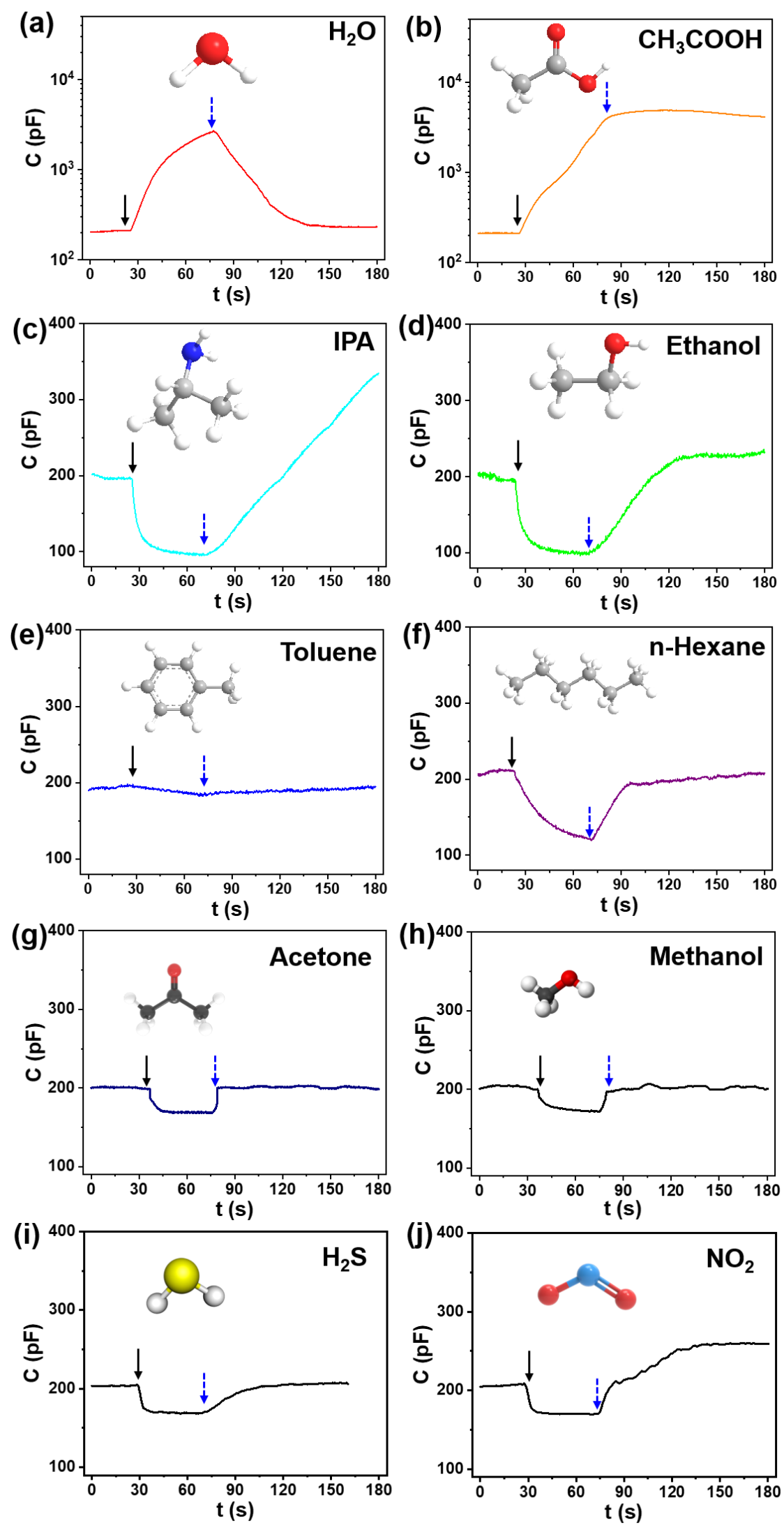

Figure S4. Capacitance response and recovery curves at $1 \mathrm{kHz}$ of $\mathrm{F}_{120} \mathrm{E}_{32} \mathrm{M}_{152}-\mathrm{PA}$ humidity sensor with (a) 12,000 ppm water, (b) 22,000 ppm acetic acid, (c)780,000 
ppm isopropyl amine, (d) 80,000 ppm ethanol, (e) 40,000 ppm toluene, (f) 200, 000 ppm hexane, (g) 300,000 ppm acetone, (h) 171,000 ppm methanol, (i) 10,000 ppm $\mathrm{H}_{2} \mathrm{~S}$, and (j) 10,000 ppm $\mathrm{NO}_{2}$. The concentration of interfering vapors is selected according to their saturated vapor pressures at given experimental conditions. $\mathrm{H}_{2} \mathrm{~S}$ and $\mathrm{NO}_{2}$ with $1 \% \mathrm{~mol} / \mathrm{mol}$ concentration are purchased and used without further dilution. The black solid arrow and blue dash arrow indicate the time point of introducing and removing vapor, respectively. The decreasing capacitance toward isopropyl amine, ethanol, hexane, acetone, Methanol, $\mathrm{H}_{2} \mathrm{~S}$ and $\mathrm{NO}_{2}$ is probably caused by their relatively lower dipole moment, dielectric constant and polarity when compared with water.
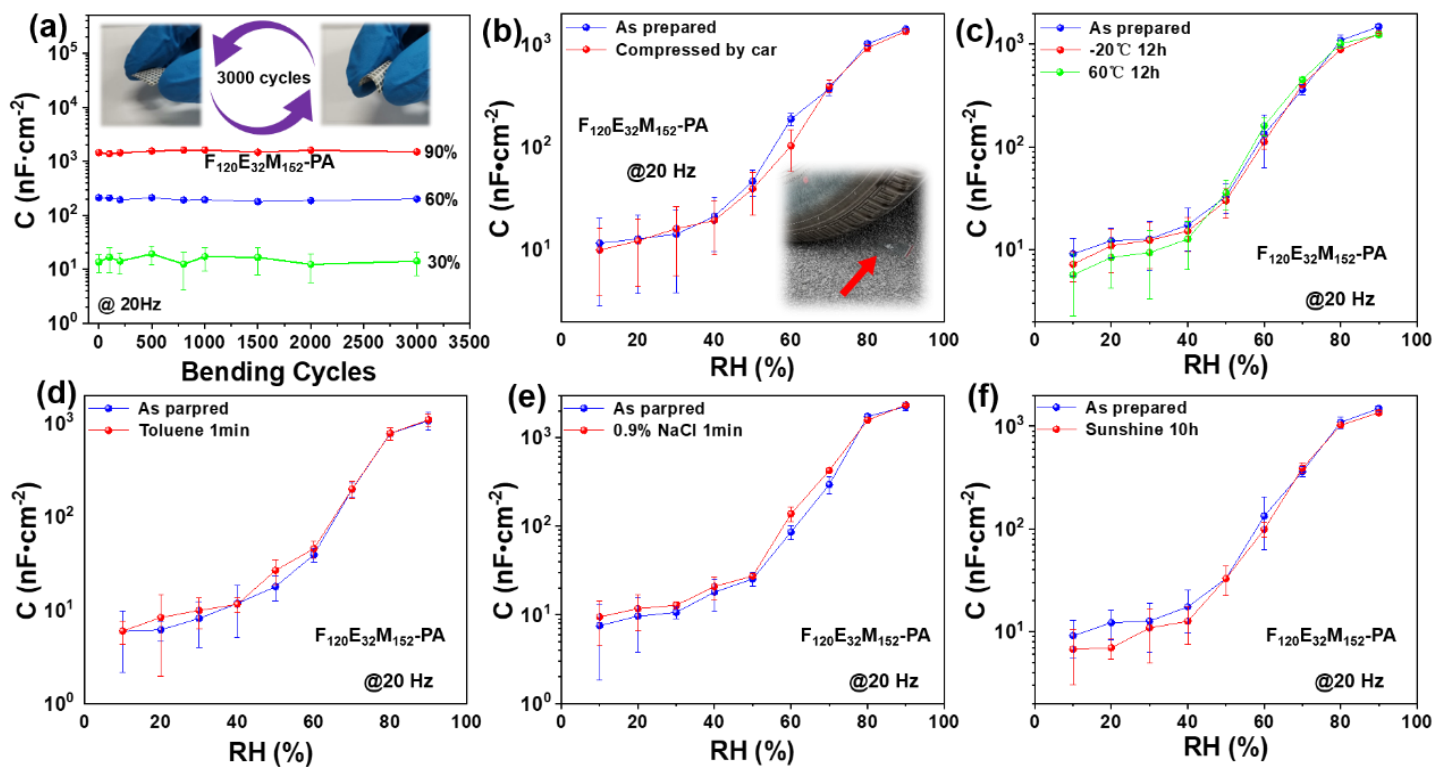

Figure S5. Capacitance change of $\mathrm{F}_{120} \mathrm{E}_{32} \mathrm{M}_{152}$-PA after (a) 3000 cycles of bending with a bending radius of $5 \mathrm{~mm}$, (b) compression by a vehicle weighting $2000 \mathrm{~kg}$, (c) experiencing high $\left(60^{\circ} \mathrm{C}\right)$ and low $\left(-20^{\circ} \mathrm{C}\right)$ temperatures, (d) toluene etching, (e) $0.9 \%$ $\mathrm{NaCl}$ aqueous solution etching and (f) intensive sun irradiation for $10 \mathrm{~h}$. 


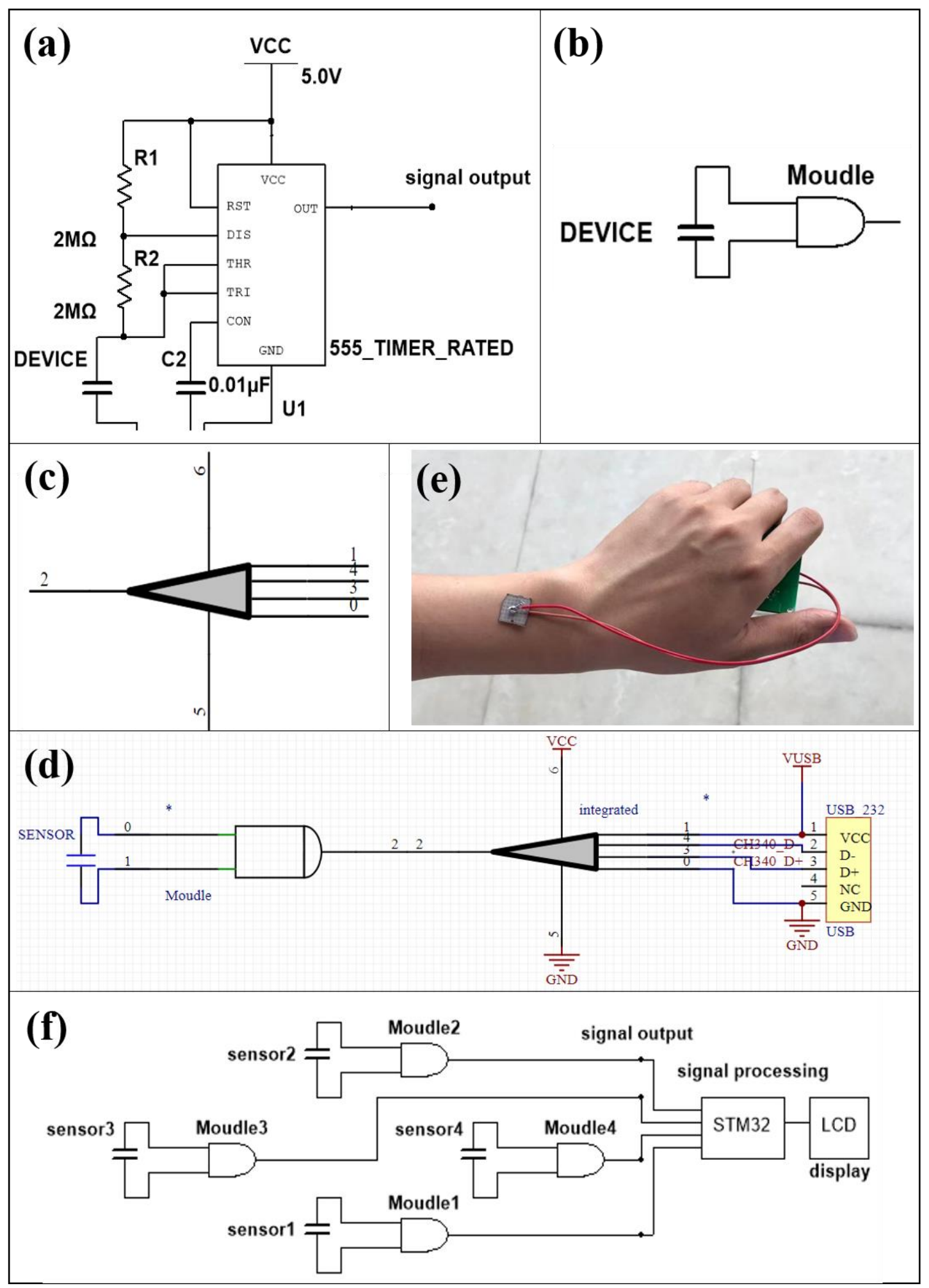

Figure S6. (a) Signal conditioning circuit for capacitance measurement; (b) Simplified view of (a); (c) Simplified view of units for data analysis circuit shown in Figure S13; (d) Circuit for the whole testing system; (e) Capacitor sensor on the arm wired to the signal conditioning circuit; (f) Signal conditioning circuit used to determine the water source location. 

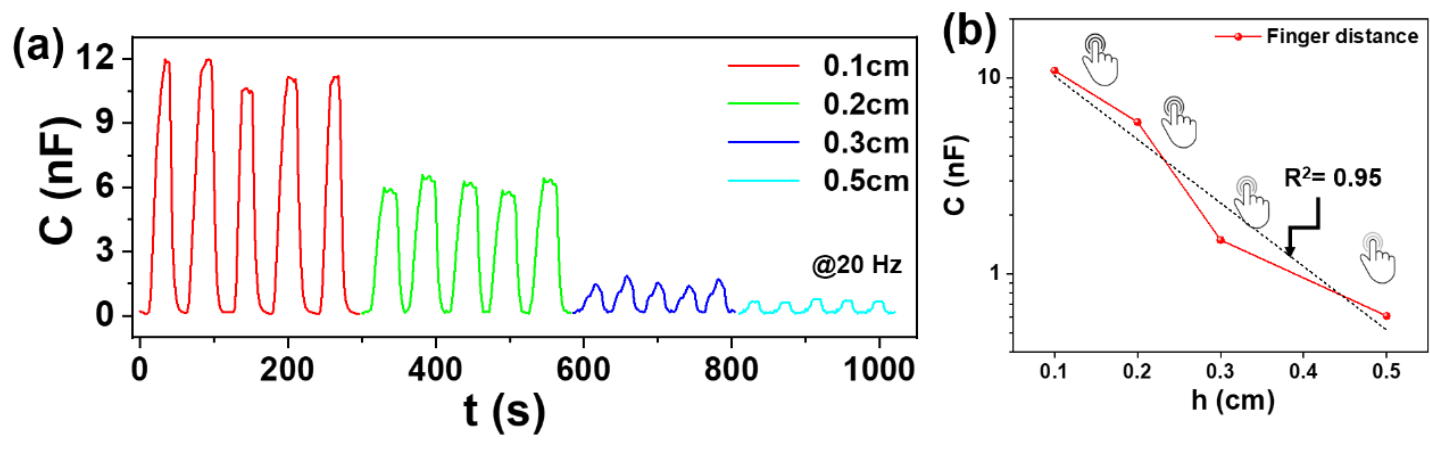

Figure S7. (a) Repeated approaching finger to sensor surface with different distances. (b) Linear fitting of the capacitance versus finger distance to the sensor surface.
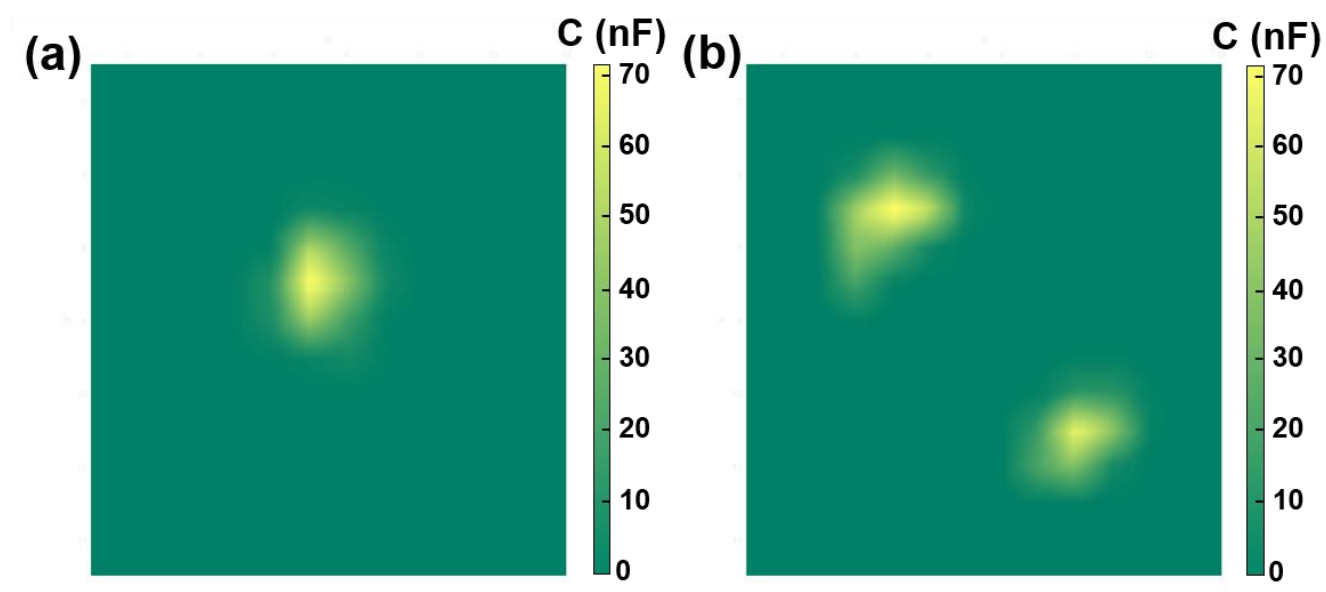

(c)
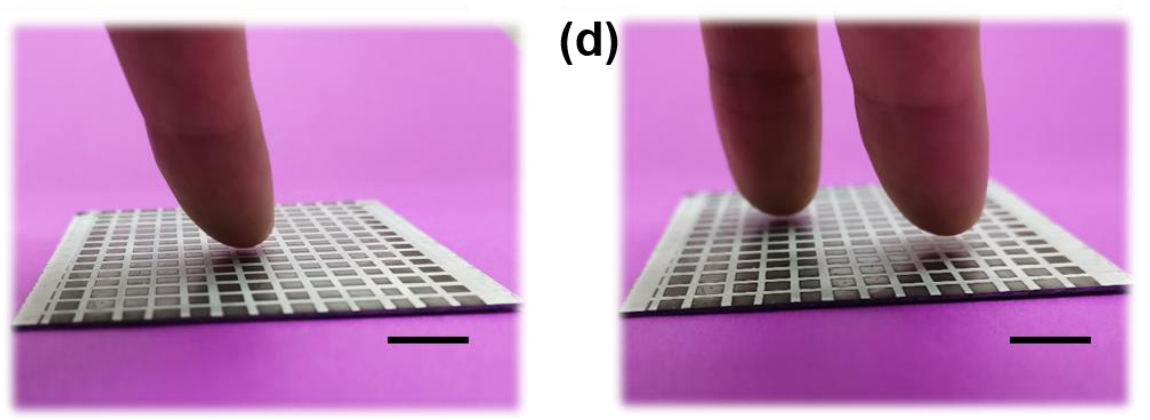

Figure S8. Perceiving finger position without direct contact by an array of humidity sensor with (a-c) one finger and (b-d) two fingers closing the sensor surface. The scale bar in $(\mathrm{c}, \mathrm{d})$ is $1 \mathrm{~cm}$.
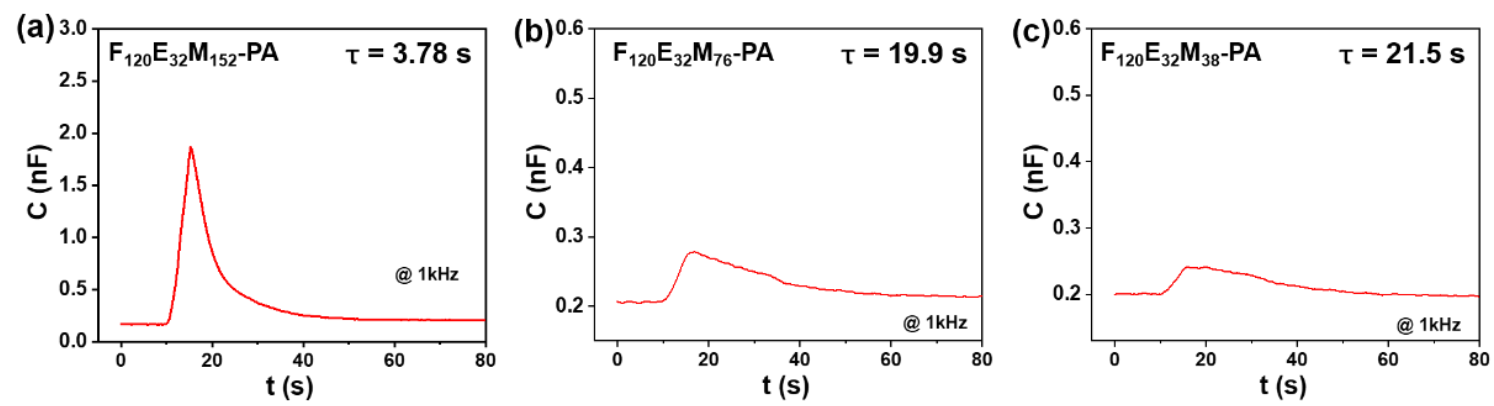

Figure S9. Decay time of films with different pore content after moisture pulse removal. 
Determining the distance " $r$ " between the water source and sensor circuit in Figure 8c-d:

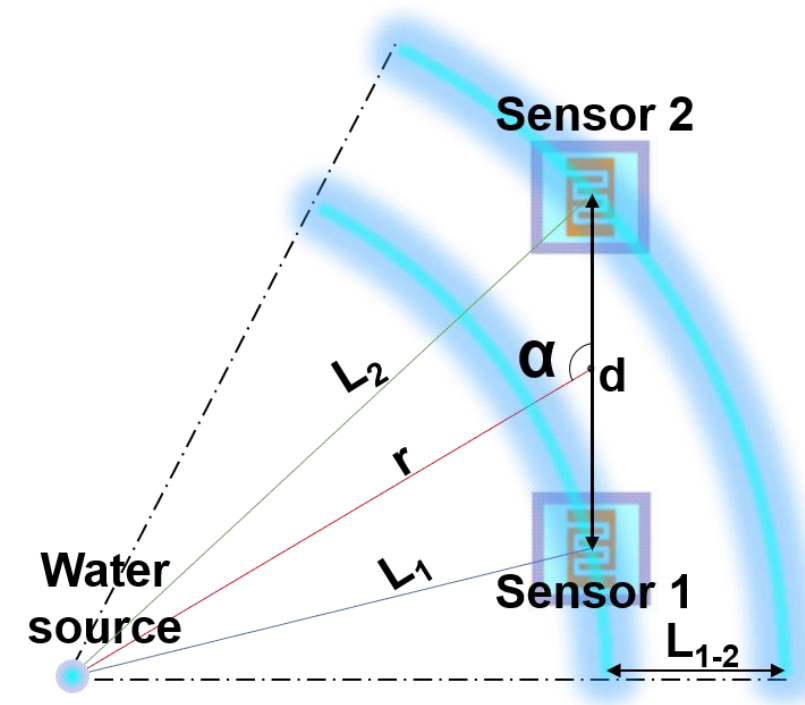

Figure S10. Geometric relationship between $L_{1}, L_{2}, r, d$ and $\alpha$.

From Figure S10, following equations could be concluded:

$$
\begin{gathered}
L_{1-2}=L_{2}-L_{1} \\
L_{2}=\sqrt{(r \sin \alpha)^{2}+\left(\frac{d}{2}-r \cos \alpha\right)^{2}} \\
L_{1}=\sqrt{(r \sin \alpha)^{2}+\left(\frac{d}{2}+r \cos \alpha\right)^{2}} \\
L_{1-2}=\sqrt{(r \sin \alpha)^{2}+\left(\frac{d}{2}-r \cos \alpha\right)^{2}}-\sqrt{(r \sin \alpha)^{2}+\left(\frac{d}{2}+r \cos \alpha\right)^{2}}
\end{gathered}
$$

Equation S1

Equation S1 could be rewritten as equation 2:

$$
r=L_{1-2} \sqrt{\frac{d^{2}-L_{1-2}{ }^{2}}{4\left(d^{2}(\cos \alpha)^{2}-L_{1-2}{ }^{2}\right)}}
$$

Equation 2

Where " $d$ " is the distance between sensor 1 and 2 , " $L_{1-2}$ " is the travel distance of moisture pulse during the period from $t 1$ to $t 2$ (Figure $8 \mathrm{c}$ ).

\section{Sensor circuit design and fabrication:}

\section{Design of Hardware Circuit}


In this work, 32-bit microprocessor STM32F103 is used as the main control unit, and its internal $\mathrm{AD}$ converter is used for signal analog-to-digital conversion. The block diagram of hardware circuits including voltage stabilized filter circuit, AD converter circuit, USB data transfer circuit, keyboard scanning and LCD display are shown in Figure S11.

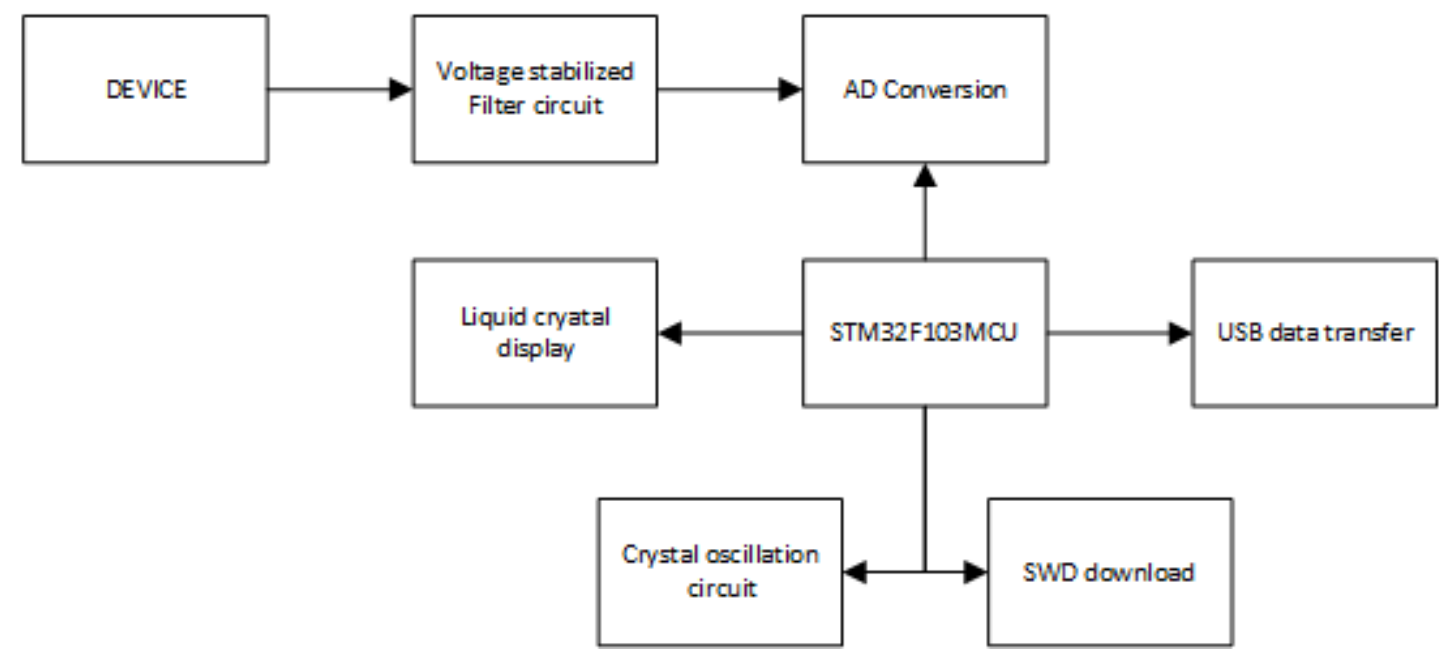

Figure S11. The block diagram of hardware.

\section{Data acquisition ADC module}

The 12-bit ADC is used as a successive approximation analog-to-digital converter. It has up to 18 multiplexed channels which could measure signals from 16 external and two internal sources. The results acquired by ADC are stored in a 16-bit data register. The analog watchdog feature makes it possible to detect the input voltage which exceed the user-defined thresholds. Figure S12 shows a single ADC diagram.

\section{ADC main features}

-12-bit resolution

- Interrupt generation at End of Conversion, End of Injected conversion and Analog

- watchdog event

- Single and continuous conversion modes

- Scan mode for automatic conversion of channel 0 to channel ' $n$ '

- Self-calibration

- Data alignment with in-built data coherency 
- Channel by channel programmable sampling time

- External trigger option for both regular and injected conversion

- Discontinuous mode

- Dual mode (on devices with 2 ADCs or more)

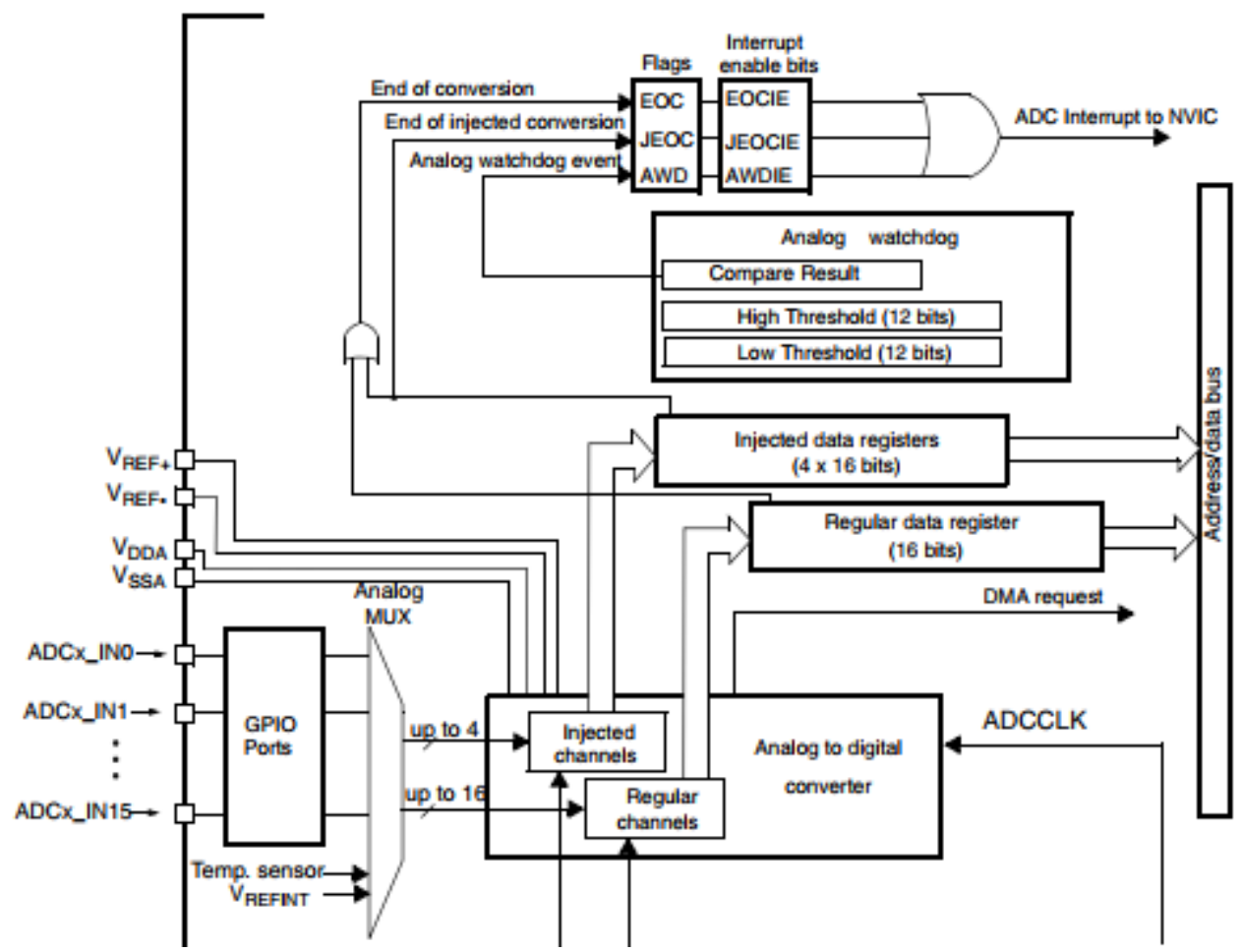

Figure S12. Single ADC diagram.

\section{Microprocessor module}

We used STM32F103Zett6 as MCU of this circuit. This chip also has an external bus (FSMC) can be used to expand SRAM and connect LCD which can significantly improve the LCD refresh speed. The external circuit schematic diagram of the main control chip F103ZET6 is shown in Figure S13. U1 is our main chip: STM32F103ZET6. $\mathrm{R} 7$ and $\mathrm{R} 8$ are isolated from the $\mathrm{MCU}$ and the external power supply which are designed for the maintenance. 


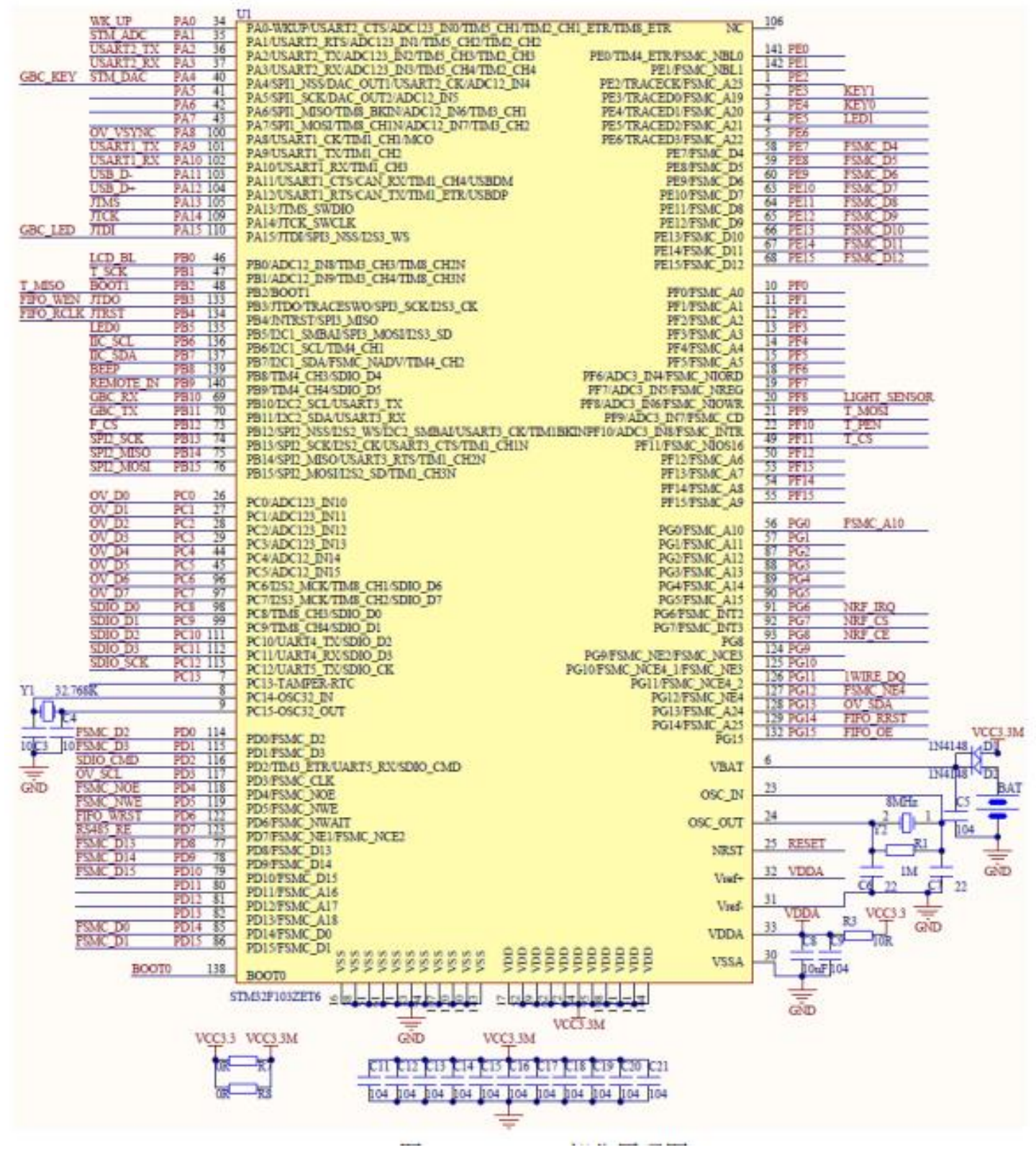

Figure S13. main control chip external circuit.

\section{Voltage stabilized filter circuit}

STM32F103ZET6 processor is a low-power processor which could switch 5V working voltage into $3.3 \mathrm{~V}$. The $0.1 \mu \mathrm{F}$ capacitor connected to the ground is mainly used to decouple and filter the peak pulse on the power supply signal. Besides, the $220 \mu \mathrm{F}$ capacitor combined with $0.1 \mu \mathrm{F}$ capacitor could makes the power signal stable and clean. The circuit diagram is shown in Figure S14. The ADC working voltage supply is also in this circuit and protect the digital power from interfering by the analog power supply. 


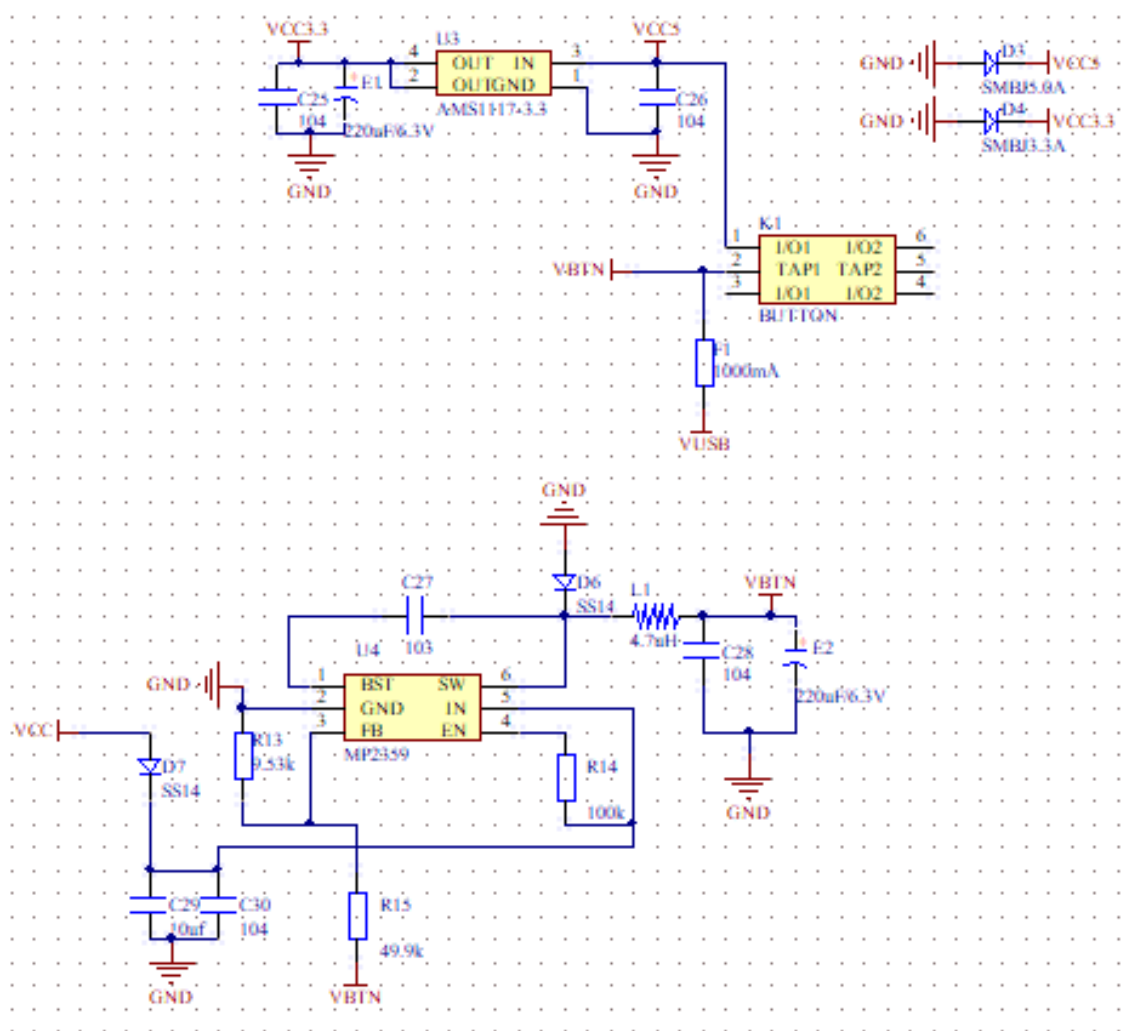

Figure S14. Voltage stabilized filter circuit.

\section{Crystal oscillation circuit}

A crystal oscillator with a frequency of $8 \mathrm{M}$ is used for the calculation of accurate time, the circuit structure is shown in Figure S15.

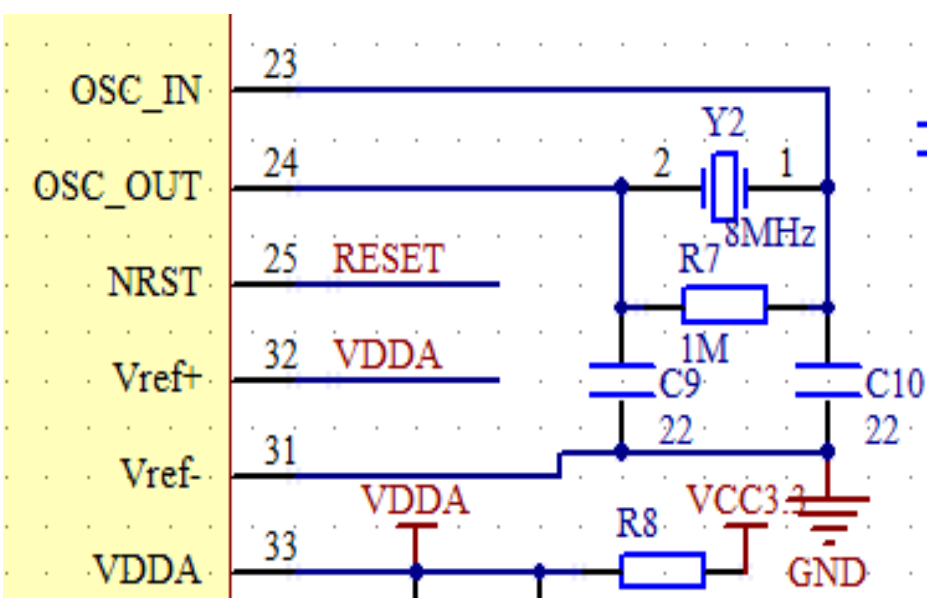

Figure S15. Crystal oscillator circuit structure diagram 
Table S1. Detail information of $\mathrm{F}_{\mathrm{x}} \mathrm{E}_{\mathrm{y}} \mathrm{M}_{\mathrm{z}}$ film with different componential ratios.

\begin{tabular}{|c|c|c|c|c|c|c|}
\hline$F_{x} E_{y} M_{z}$ & \multirow{2}{*}{$\begin{array}{c}b P E I \\
/ \text { pPFPA }^{a}\end{array}$} & \multirow{2}{*}{$\mathrm{PMMA} /(\mathrm{pPFPA}+b \mathrm{PEI})^{\mathrm{b}}$} & \multirow{2}{*}{$\begin{array}{c}\text { pPFPA (10 } \\
\mathrm{mg} / \mathrm{ml})\end{array}$} & \multirow{2}{*}{$\begin{array}{c}b \text { PEI (10 } \\
\mathrm{mg} / \mathrm{ml})\end{array}$} & \multirow{2}{*}{\multicolumn{2}{|c|}{ PMMA }} \\
\hline$x: y: z$ & & & & & & \\
\hline $120: 32: 0$ & $50 \%$ & $0 \%$ & $120 \mu \mathrm{l}$ & $32 \mu \mathrm{l}$ & & 0 \\
\hline $120: 32: 38$ & $50 \%$ & $25 \%$ & $120 \mu \mathrm{l}$ & $32 \mu \mathrm{l}$ & $15 \mu 1$ & $25 \mathrm{mg} / \mathrm{ml}$ \\
\hline $120: 32: 76$ & $50 \%$ & $50 \%$ & $120 \mu \mathrm{l}$ & $32 \mu \mathrm{l}$ & $30 \mu \mathrm{l}$ & $25 \mathrm{mg} / \mathrm{ml}$ \\
\hline $120: 32: 152$ & $50 \%$ & $100 \%$ & $120 \mu 1$ & $32 \mu \mathrm{l}$ & $30 \mu 1$ & $50 \mathrm{mg} / \mathrm{ml}$ \\
\hline 120:16:136 & $25 \%$ & $100 \%$ & $120 \mu \mathrm{l}$ & $16 \mu \mathrm{l}$ & $27 \mu \mathrm{l}$ & $50 \mathrm{mg} / \mathrm{ml}$ \\
\hline $120: 48: 168$ & $75 \%$ & $100 \%$ & $120 \mu \mathrm{l}$ & $48 \mu \mathrm{l}$ & $33 \mu \mathrm{l}$ & $50 \mathrm{mg} / \mathrm{ml}$ \\
\hline
\end{tabular}

a) Molar ratio, b) weight ratio

Table S2. Thicknesses of FxEyMz, FxEyMz-P and FxEyMz-PA, respectively.

\begin{tabular}{|c|c|c|c|c|c|}
\hline $\mathbf{F}_{\mathbf{x}} \mathbf{E}_{\mathbf{y}} \mathbf{M}_{\mathrm{z}}$ & \multirow{2}{*}{$\begin{array}{c}\text { Thickness } \\
\qquad(\mu \mathrm{m})\end{array}$} & $\mathbf{F}_{\mathrm{x}} \mathbf{E}_{\mathbf{y}} \mathbf{M}_{\mathbf{z}}-\mathbf{P}$ & \multirow{2}{*}{$\begin{array}{c}\text { Thickness } \\
(\mu \mathrm{m})\end{array}$} & $\mathbf{F}_{\mathbf{x}} \mathbf{E}_{\mathbf{y}} \mathbf{M}_{\mathrm{z}}-\mathbf{P A}$ & \multirow{2}{*}{$\begin{array}{c}\text { Thickness } \\
(\mu \mathrm{m})\end{array}$} \\
\hline$x: y: z$ & & $x: y: z$ & & $x: y: z$ & \\
\hline $120: 32: 0$ & 3.35 & $120: 32: 0$ & 2.23 & $120: 32: 0$ & 1.69 \\
\hline $120: 32: 38$ & 3.59 & $120: 32: 38$ & 2.37 & $120: 32: 38$ & 1.27 \\
\hline $120: 32: 76$ & 4.25 & $120: 32: 76$ & 1.69 & $120: 32: 76$ & 1.50 \\
\hline $120: 32: 152$ & 6.17 & $120: 32: 152$ & 2.22 & $120: 32: 152$ & 1.74 \\
\hline 120:16:136 & 8.74 & $120: 16: 136$ & 0.74 & $120: 16: 136$ & 0.67 \\
\hline $120: 48: 168$ & 9.90 & $120: 48: 168$ & 2.96 & $120: 48: 168$ & 2.07 \\
\hline
\end{tabular}

Table S3. Error of locating capability.

\begin{tabular}{|c|c|c|c|}
\hline Parameter & $\alpha$ & $\beta$ & $r$ \\
\hline \multirow[t]{2}{*}{ Error } & $\pm 5^{\circ}$ & $\pm 5^{\circ}$ & $\leqslant 5 \%(r<25 \mathrm{~cm})$ \\
\hline & & & $\leqslant 15 \% \quad(25 \mathrm{~cm}<r<50 \mathrm{~cm})$ \\
\hline
\end{tabular}

Each data point is a standard deviation from at least five individual experiments. If the distance between the water source and sensor circuit becomes much longer, the time interval when the moisture pulse passes through four individual sensors, i.e., $\Delta t_{1-2}$ and $\Delta t_{3-4}$, only show slight changes, which are beyond the time resolution of data acquisition of the CPU chip. As a result, the locating capability is weakened. Improving the data acquisition rate (time resolution) of the CPU chip would help to reduce the error of locating capability.

\section{References:}

(1) Johnson, E. R.; Keinan, S.; Mori-Sánchez, P.; Contreras-García, J.; Cohen, A. J.; Yang, W, Revealing Noncovalent Interactions. J. Am. Chem. Soc. 2010, 132, 6498-6506. (2) Neese, F. Software Update: The ORCA Program System, version 4.0, Wiley: 
Germany, 2017, WIREs Comput Mol Sci 2017, e1327. doi: 10.1002/wcms.1327.

(3) Grimme S, Antony J, Ehrlich S, Krieg H. A Consistent and Accurate ab initio Parametrization of Density Functional Dispersion Correction (DFT-D) for the 94

Elements H-Pu. J. Chem. Phys. 2010, 132, 154104-154119.

(4) Weigend, F.; Ahlrichs, R. Balanced Basis Sets of Split Valence, Triple zeta Valence and Quadruple zeta Valence Quality for H to Rn: Design and Assessment of Accuracy. Phys. Chem. Chem. Phys. 2005, 7, 3297-3305.

(5) Emamian, S.; Lu, T.; Kruse, H.; Emamian, H., Exploring Nature and Predicting Strength of Hydrogen Bonds: A Correlation Analysis between Atoms-In-Molecules Descriptors, Binding Energies, and Energy Components of Symmetry-Adapted Perturbation Theory. J. Comput. Chem. 2019, 40 (32), 2868-2881. 